\title{
Towards a Short-range Free-space GHz-clocked Quantum Key Distribution System
}

\author{
M. J. García-Martínez, N. Denisenko, D. Soto, V. Fernández \\ Institute of Applied Physics, Spanish National Research Council (CSIC), \\ Serrano 144, 28006 Madrid, Spain \\ mariajose.garcia@iec.csic.es
}

\begin{abstract}
A free-space quantum key distribution system that operates at a wavelength of $850 \mathrm{~nm}$ is presented. The system is designed to implement the B92 protocol at high transmission rates between two locations in urban areas.

OCIS codes: (270.5568) Quantum cryptography; (060.2605) Free-space optical communication
\end{abstract}

\section{Introduction}

Quantum key distribution (QKD) uses physical properties of single photons, such as polarization or phase, to securely distribute a cryptographic key between two parties [1]. The security of QKD is guaranteed by the laws of quantum mechanics, and it is currently the only means to securely exchange cryptographic keys by detecting the presence of an eavesdropper in the channel.

QKD has greatly evolved since the first experimental demonstration in 1992 [2] and distances up to $144 \mathrm{~km}$ in free space [3] and $250 \mathrm{~km}$ in optical fiber [4] have successfully been achieved. However, due to both the impossibility of using quantum repeaters and employing weak coherent pulses (WCP) instead of true single photon sources, key exchange rates of current QKD systems are still considerably low. Free-space QKD systems can make use of the spectral region around $850 \mathrm{~nm}$ where the atmosphere, unlike optical fiber, presents a low absorption window. At this wavelength silicon single-photon avalanche diode (Si-SPAD) detectors can be used, which can be operated at high frequencies allowing higher transmission rates.

Moreover, free-space optics offers the possibility of establishing earth-to-satellite links which would allow global QKD. For shorter-ranges scenarios, conventional Free Space Optics (FSO) is alleviating the connectivity bottleneck by the installation of free-space optical links in critical points of fibre-optic metropolitan networks where the bandwidth is severely diminished due to the poor - or lack of - connections of some parts of the network to the backbone. QKD applied to these links would offer both security and speed, and would be attractive for commercial and financial buildings that wish to be securely connected to the backbone network without the need of the expensive licenses and high cost generated from installing optical fiber.

We are thus building a GHz-clocked free-space QKD system that implements the $\mathrm{B} 92$ protocol [5] at GHz clock frequencies between two locations in Madrid. The system will be described in detail in the next section.

\section{Description and characterization of the experimental system}

As mentioned above the system we are building implements the B92 protocol, which uses two non-orthogonal polarization states to encrypt the binary values ' 1 ' and ' 0 '. Figure 1 shows the layout of the transmitter optics.

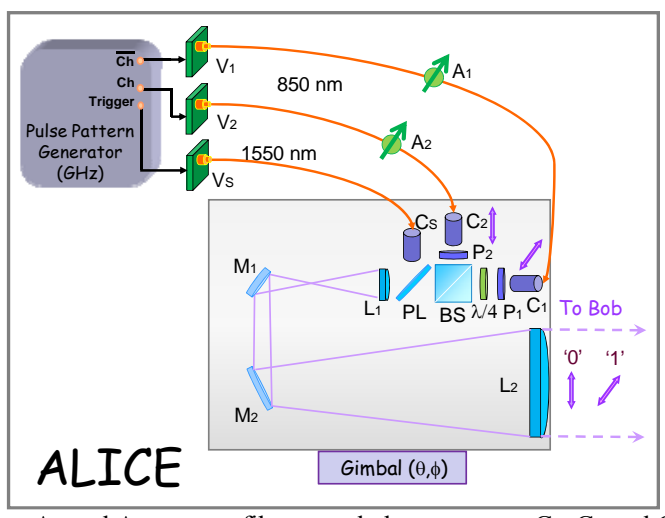

Fig 1. $V_{1}$ and $V_{2}$ are two VCSELs at $850 \mathrm{~nm} ; A_{1}$ and $A_{2}$ are two fiber-coupled attenuators; $C_{1}, C_{2}$ and $C_{S}$ are three beam collimators; $P_{1}$ and $P_{2}$ are two high-extinction-ratio polarizers; $\mathrm{BS}$ is a cube beam-splitter; $\mathrm{L}_{1}$ and $\mathrm{L}_{2}$ are two achromatic lenses that expand and collimate the beam; $\mathrm{M}_{1}$ and

$\mathrm{M}_{2}$ are two high-reflectivity mirrors. $\mathrm{V}_{\mathrm{S}}$ is a third VCSEL at $1550 \mathrm{~nm}$ used for the synchronization of transmitter and receiver and is combined with the laser beam at $850 \mathrm{~nm}$ by using a wide spectrum pellicle (PL). 
To achieve high transmission rates Alice uses two vertical-cavity surface emitting lasers (VCSELS) at a wavelength of $\lambda \sim 850 \mathrm{~nm}$, which are controlled by high-speed drivers. The lasers are modulated by a fast $\mathrm{GHz}$ pre-programmed pulse pattern generator able to generate pseudo-random sequences up to $2^{31}-1$ bits. The output of each laser is then collimated and polarized by two high-extinction-ratio polarizers to generate the two polarization states required by the B92 protocol. These states are then combined by a non-polarizing beam splitter cube and the beam is then expanded to a diameter of $\sim 40 \mathrm{~mm}$ using a set of two achromatic lenses. A synchronization timing signal is emitted at a different wavelength $(1550 \mathrm{~nm})$ and it is combined with the output beam by using a broadband pellicle beam splitter. The transmitter is mounted on a high-precision gimbal system, which will facilitate the alignment and pointing with the receiver. It consists of a structure which provides with the movements of right ascension and declination with precision of microradians.

The receiver will be located at about $3 \mathrm{~km}$ from the transmitter. Its layout is shown in figure 2 . The beam coming from Alice is focused by using a Cassegrain telescope with $25.4 \mathrm{~cm}$ of diameter aperture. The receiver optics has been designed to be directly coupled to the output of the telescope. As the optical signal reaches the receiver, the 'data' beam at $850 \mathrm{~nm}$ and the 'synchronization' beam at $1550 \mathrm{~nm}$ are separated by a dichroic mirror. The solar background radiation is filtered by a band-pass spectral filter at $850 \mathrm{~nm}$ in conjunction with the spatial filtering carried out by the optical fiber and additional software processes. The states ' 1 ' and ' 0 ', encrypted by the two non-orthogonal polarization states, are discriminated in the receiver by using a non-polarizing beamsplitter and two high-extinction polarizers. Two $\lambda / 4$ and one $\lambda / 2$ wave plates are also used to recover the linearity of the polarization states. The photons will be detected by two silicon Single-Photon Avalanche Diodes (SPADs) and a high speed Time Interval Analyzer (TIA) measures the arrival time of each of the photons. These arrival times serve to reconstruct the signal sent by Alice and determine the security of the transmission, i.e. the quantum bit error rate.

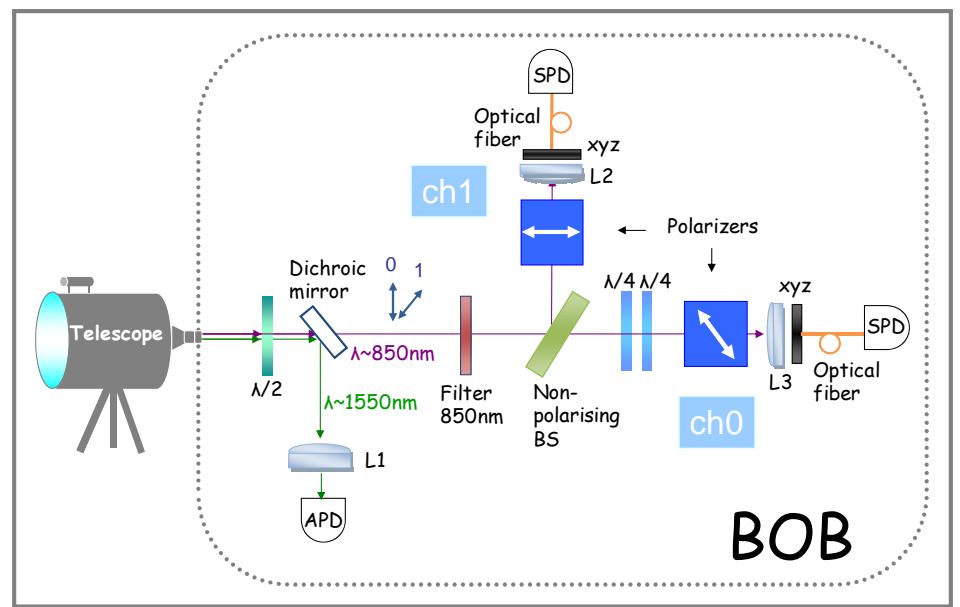

Fig 2. Setup of the receiver. The 'synchronization' and 'data' signals are separated by a dichroic mirror; a $\lambda / 2$ wave plate is used to correct the angle of the polarization states; the solar radiation is spectrally filtered by a band-pass filter centered at $850 \mathrm{~nm}$ and spatially by the optical fibers; the two non-orthogonal polarizations states are distinguished by a non-polarizing beam-splitter, two polarizers and two single photon detectors; two $\lambda / 4$ wave plates improve the linearity of the polarization states; and an avalanche photodetector is used to detect the synchronization signal.

The QKD system has been characterized in terms of polarization and losses and an estimation of the Quantum Bit Error Rate (QBER) has also been measured. The polarization extinction ratio (PER) of the diagonal linearly polarized state exiting Alice decreases after being reflected by the two mirrors. These mirrors have a metallic coating, which makes the index of refraction complex, adding a phase shift between both components of the electric field (parallel and perpendicular to the incidence plane). This effect does not occur for the vertical state as the electric field of the polarized state can be contained in only one of both planes. The linearity of the diagonal state is corrected by a quarter waveplate at the transmitter before it is combined with the vertical state. At the receiver the polarization also gets degraded due to the birefringence of certain optical components. Therefore a half waveplate and two extra quarter waveplates are inserted to correct for it. The error improves after the correction from $4 \%$ to $1.4 \%$.

Also the transmission efficiency of the receptor has been characterized and is around $40 \%$. However there is an extra $60 \%$ loss due to the coating of the telescope not being designed for our operating wavelength.

In the most coming future, several tests of the system at various distances between 0 and $3 \mathrm{~km}$ will be performed. Moreover an automatic tracking process with high-precision motors will be implemented so that relative 
deviations between both stations due to expansion and compression of buildings or turbulence in the atmosphere can be corrected. For that purpose two PCs will control the tip/tilt movements of the transmitter and receiver's gimbals with the feedback information provided by two position sensitive devices (PSD) in the transmitter and receiver. The PSDs will detect any deviation from the position of a beacon laser shining between transmitter and receiver and parallel to the data beam and will order the motors to compensate for them.

\section{Conclusions}

A high-bit-rate free-space QKD system for urban-span applications has been presented. The modulation of highbandwidth laser diodes with a fast frequency generator in Alice, in conjunction with an optical synchronization at a different wavelength and detectors in Bob that can be operated at high frequencies, will permit faster key generation than those currently achieved. In addition, the polarization extinction ratio of the quantum states has been measured and corrected to provide an improvement on the estimated QBER due to polarization leakage of $65 \%$. Finally, a tracking scheme that will allow continuous operation of the proposed QKD system is being currently studied.

\section{Acknowledgements}

We would like to thank the Ministerio de Educación y Ciencia, projects MTM2008-02194 and 200950I073, and CDTI, Ministerio de Industria, Turismo y Comercio (Spain), in collaboration with Telefónica I+D, project SEGUR@with reference CENIT-2007 2004.

\section{References}

[1] Gisin N., Ribordy G., Tittel W. and Zbinden H., "Quantum cryptography”, Rev. Mod. Phys. 74 145-195 (2002).

[2] Bennett C. H., Bessette F., Brassard G., Salvail L. and Smolin J., “Experimental quantum cryptography”, J. Crypto.5 3-28 (1992).

[3] T. Schmitt-Manderbach et al. "Experimental demonstration of free-space decoy-state quantum key distribution over 144 km", Physical Review Letters 98, 010504 (2007).

[4] D Stucki, N Walenta, F Vannel, R T Thew, N Gisin, H Zbinden, S Gray, C R Towery and S Ten, "High rate, long-distance quantum key distribution over $250 \mathrm{~km}$ of ultra low loss fibres”, New J. Phys. 11075003 (2009).

[5] C. H. Bennet, "Quantum cryptography using any two nonorthogonal states”, Physical Review Letters 68, 3121 (1992). 